\title{
MODELLING AND SIMULATION OF A NEW VARIABLE STIFFNESS HOLDER FOR MILLING OF FLEXIBLE DETAILS
}

\author{
Krzysztof J. Kaliński*, Marek A. Galewski', Michał Mazur*, Marek Chodnicki
}

"Faculty of Mechanical Engineering, Gdańsk University of Technology, Poland

\begin{abstract}
Modern industry expectations in terms of milling operations often demand the milling of the flexible details by using slender ball-end tools. This is a difficult task because of possible vibration occurrence. Due to existence of certain conditions (small depths of cutting, regeneration phenomena), cutting process may become unstable and self-excited chatter vibration may appear. Frequency of the chatter vibration is close to dominant natural frequency of the workpiece or the tool. One of the methods of chatter vibration avoidance is matching the spindle speed to the optimum phase shift between subsequent cutting edges passes (i.e. the Liao-Young condition). However, the set of optimum spindle speeds from the point of view of vibration reduction may be not optimum one from other points of view. For example milling efficiency or machine tool capabilities cannot be assured. This article presents the idea of a workpiece holder with adjustable stiffness and discusses a new variant of its realization. In the holder, milling process is performed at constant spindle speed and feed speed. In order to avoid vibration the holder stiffness is modified. Stiffness changes modify natural frequencies of the workpiece and thus it is possible to modify dynamic properties of the workpiece in such a way that arbitrary chosen, constant spindle speed will be optimum, due to the Liao-Young condition performance. Calculation of the optimum stiffness is performed before milling, based on the workpieces modal identification results and the finite element model simulations.
\end{abstract}

Keywords: milling, vibration reduction, optimum spindle speed, optimum stiffness

\section{INTRODUCTION}

Modern industry expectations in terms of milling operations often demand the milling of the flexible details (i.e. offshore structures, rotor blades, thin-walled elements) by using slender ball-end tools. This is demanding task because it is usually required to achieve surface quality high enough to treat milling operation final without the required additional finishing operations. Milling flexible details is also a challenging problem because such objects may be prone to vibration especially when the workpiece is difficult to support or cannot be supported during milling processes. In such case, tool-workpiece relative vibration plays a principal role because, in certain conditions, it may lead to loss of stability and generation of self-excited chatter vibration (Tomków, 1997). Additionally, vibrations in a machine tool system do not only reduce surface quality but also increase tool wear and, in extreme cases, may lead to the destruction of a tool or a workpiece (Nouari et al., 2003).

The regeneration phenomenon is recognised the most important reason for chatter vibration occurrence (Quintana and Ciurana, 2011). There are many different methods for reduction and surveillance of the chatter vibration, i.e. the use of cutting edge chamfers (Sellmeier and Denkena, 2012), using mechanical dampers (Kim et al., 2006) or smart materials (Rashid, 2005), robust optimum control (Moradi et al., 2013), active methods (i.e. active structural control (Dohner et al., 2004), active holder (Brecher et al., 2010), active damping (Ganguli et al., 2005; Parus et al., 2009; Kaliński and Galewski, 2014)), cutting with variable spindle speed (Soliman and Ismail, 1997; Kaliński and Galewski, 2011).

Another method depends on matching the spindle speed to the optimum phase shift between subsequent passes of the tool cutting edges, which was proposed by Liao and Young (Liao and Young, 1996). This optimum spindle speed can be 
calculated by using the generalised Liao-Young condition (Kaliński and Galewski, 2015):

$$
\frac{z n_{\alpha}}{60}=\frac{f_{\alpha}}{0.25+m}, \quad m=0,1,2, \ldots,
$$

where:

$f_{\alpha}$ - workpiece natural frequency of no. $\alpha[\mathrm{Hz}]$,

$n_{\alpha}$ - requested spindle speed [rev/min],

$z$ - number of cutting edges of the milling tool.

The only unknown element in Eq. 1 is dominant natural frequency $f_{\alpha}$ which may be identified, for example, by performing the modal test. As the result, a set of optimum spindle speeds is obtained. From this set, one speed is selected for performance of milling operation. However, the optimum spindle speed from the point of view of the Liao-Young condition, may not be optimum from the point of view of the other requirements, i.e. cutting process productivity. For example, if $f_{\alpha}=166,6 \mathrm{~Hz}$ and $z=2$, the set of optimum spindle speeds is $n_{\alpha} \in<2000,4000,2222,1538, \ldots>[\mathrm{rev} /$ min]. A large gap between the first and the second speed can be observed. This may lead to non-optimum utilization of a milling centre potential. For example, when milling centre can achieve speeds only up to $18000 \mathrm{rev} / \mathrm{min}$, the highest optimum spindle speed which can be selected from the calculated set, is $4000 \mathrm{rev} / \mathrm{min}$.

Moreover, complex-shaped workpieces may have different dominant natural frequencies for different workpiece zones. Thus, different spindle speeds are optimum for various workpiece areas. This leads to the idea of creating the map of optimum spindle speeds, which is described in details in (Kaliński et al., 2010; Kaliński et al., 2014a; Kaliński et al., 2013) together with some examples of successful application. However, changing spindle speed during tool pass may reduce surface quality in speed change point and is difficult to perform in some milling centres due to the properties of control units. This concerns most of all milling centres equipped with controllers which do not allow speed changes without stopping the tool or the workpiece feed. This problem can be avoided by milling a whole tool pass with only one spindle speed. However, this also means that in some zones this speed will not be optimum. Unfortunately, the map of optimum spindle speeds does not solve the problem of incongruity of optimum spindle speeds set and accessible speed range of available machine tool.

\section{A CONCEPT OF A NEW VARIABLE STIFFNESS HOLDER}

Optimal spindle speed depends on dominant natural frequency of the workpiece (Eq. 1). The frequency depends on dynamic properties of workpieces. During milling, the workpiece is mounted in a holder which is usually considered a rigid support. In one of the previous approaches the authors proposed to introduce a holder with one degree of freedom (DOF) and adjustable stiffness. The holder had a form of
1- DOF vertical column (Kaliński et al., 2014b; Kaliński et al., 2014c). Purpose of this paper is to investigate another concept of the holder whose behaviour is based on 1- DOF pivot joint (Fig. 1).

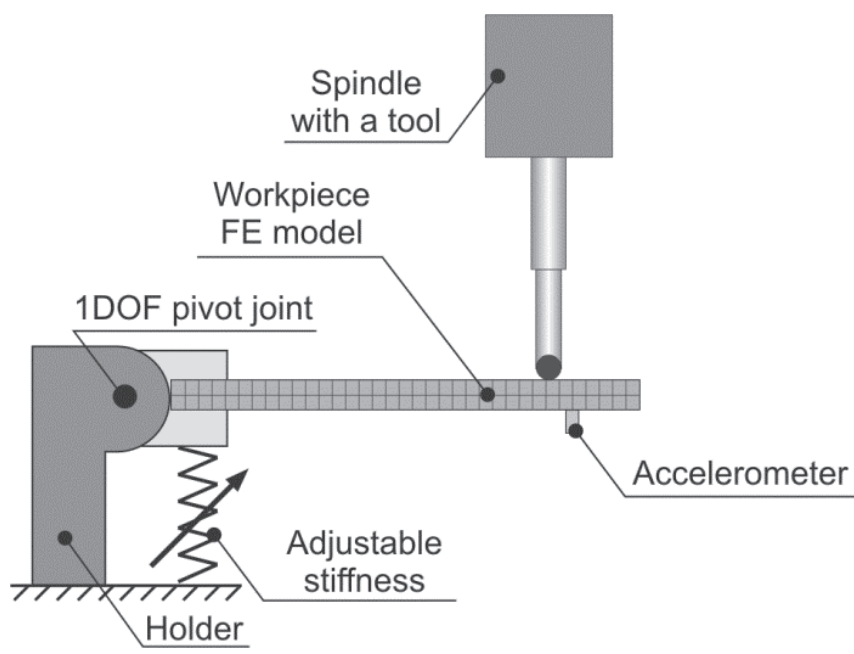

Fig. 1. Workpiece holder with adjustable stiffness and 1- DOF pivot joint and the FEM model of the workpiece

Thanks to the adjustable stiffness it is possible to modify dynamic properties of the whole system (consisting the holder and the workpiece) and to modify its natural frequency. This enables a possibility to "reverse" the current procedure of optimum spindle speed selection. Instead of calculating optimum spindle speed based on natural frequency it is possible to arbitrary set spindle speed and tune natural frequency in such a way that chosen spindle speed is optimum one, in accordance with Eq.1. For example, choosing $n_{\alpha}=$ $10000 \mathrm{rev} / \mathrm{min}$ implies that natural frequency should be one of the set : $\left.f_{\alpha} \in<83,416,750, \ldots\right\rangle[\mathrm{Hz}]$. This is achieved by suitable adjusting holder stiffness.

\section{HOLDER, WORKPIECE AND CUTTING PROCESS MODELS}

Determination of relationships between instantaneous cutting layer geometry and the resultant cutting forces became a subject of various scientific research. For example, the latter concerned identification of cutting forces for end milling (Hoffmann et al., 2010) and multi-edge milling (Pajor et al., 2011), or experimental determination of nonlinear cutting process parameters in turning (Powałka et al., 2009). For purpose of the present consideration, cutting process dynamics can be described by using the updated proportional model (Kaliński and Galewski, 2015). Based on this model, the cutting force components for edge no. $l$ depend proportionally on the instantaneous cutting layer thickness $h_{l}(t)$ and also the instantaneous depth of cutting $a_{l}(t)$; both of them vary in time. It is assumed that the resultant cutting force lies in the orthogonal plane. In accordance with directions of the action, we separate the cutting force component $F_{y l l}$ acting along nominal cutting velocity, and the cutting force component 
$F_{y 12}$ acting along cutting layer thickness (Fig. 2). The third cutting force component $F_{y l 3}$ is neglected.

$$
\begin{gathered}
F_{y l l}(t)=\left\{\begin{array}{cl}
k_{d l} a_{l}(t) h_{l}(t), & a_{l}(t)>0 \wedge h_{l}(t)>0, \\
0, & a_{l}(t) \leq 0 \vee h_{l}(t) \leq 0,
\end{array}\right. \\
F_{y l l}(t)=\left\{\begin{array}{cl}
\mu_{l} k_{d l} a_{l}(t) h_{l}(t), & a_{l}(t)>0 \wedge h_{l}(t)>0, \\
0, & a_{l}(t) \leq 0 \vee h_{l}(t) \leq 0,
\end{array}\right. \\
F_{y l 3}(t)=0,
\end{gathered}
$$

where: $a_{l}(t)=a_{p l}(t)-\Delta a_{p l}(t), h_{l}(t)=h_{D l}(t)-\Delta h_{l}(t)+\Delta h_{l}\left(t-\tau_{l}\right)$, $h_{D l}(t) \cong f_{z} \cos \varphi(t)$,

$a_{p l}(t)$ - desired depth of cutting, $\Delta a_{p l}(t)$ - dynamic change in depth of cutting, $h_{D l}(t)$ - desired cutting layer thickness; $\Delta h_{l}($.$) - dynamic change in cutting layer thickness, k_{d}$ - average dynamic specific cutting pressure, $\mu_{l}$ - cutting force ratio (quotient of forces $F_{y l 2}$ and $F_{y l 1}$ ), $t$ - time-delay between the same position of subsequent tool cutting edges, $f$ - feed per edge, $\phi_{l}(t)$ - current immersion angle of edge no. $l$.

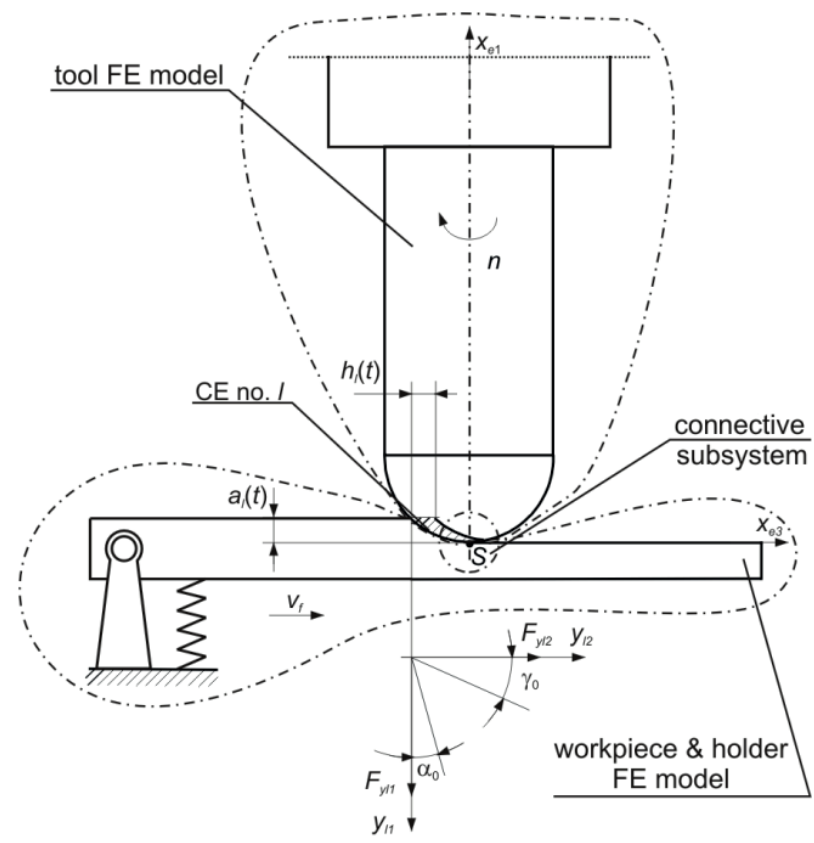

Fig. 2. Scheme of a slender ball-end milling of one-side-supported flexible workpiece in a 1-DOF pivot joint

Dynamic analysis of a slender ball- end milling process has been performed, based upon the following assumptions (Kaliński and Galewski, 2015; Kaliński, 2012):

- the spindle together with the tool fixed in the holder, and the table with the workpiece, are separated from the machine tool structure;

- only flexibility of the tool and flexibility of the workpiece are considered;

- coupling elements (CEs) are applied for modelling the cutting process dynamics;
- the effect of first pass of the edge along cutting layer causes proportional feedback, and the effect of multiple passes causes delayed feedback, additionally.

Relationships (2)...(4) describe cutting forces of CE no. $l$ in case of the proportional model. They can be presented by using matrix notation. Hence, if we neglect nonlinear geometry change components in general matrix description (Kaliński and Galewski, 2015), appropriate vector of forces of CE no. $l$ will have the following form:

$$
\begin{aligned}
\left\{\begin{array}{c}
F_{y l 1} \\
F_{y l 2} \\
F_{y l 3}
\end{array}\right\} & \underbrace{\left[\begin{array}{c}
k_{d l} a_{p l}(t) h_{D l}(t) \\
\mu_{l} k_{d l} a_{p l}(t) h_{D l}(t) \\
0
\end{array}\right]}_{\breve{\mathbf{F}}_{l}(t)}-\underbrace{\left[\begin{array}{ccc}
0 & k_{d l} a_{p l}(t) & k_{d l} h_{D l}(t) \\
0 & \mu_{l} k_{d l} a_{p l}(t) & \mu_{l} k_{d l} h_{D l}(t) \\
0 & 0 & 0
\end{array}\right]}_{\breve{\mathbf{F}}_{l}^{0}(t)} . \\
& \cdot \underbrace{\left[\begin{array}{c}
q_{z l}(t) \\
\Delta h_{l}(t) \\
\Delta a_{p l}(t)
\end{array}\right]}_{\Delta \breve{\mathbf{w}}_{P l}(t)}+\underbrace{\left[\begin{array}{ccc}
0 & k_{d l} a_{p l}(t) & 0 \\
0 & \mu_{l} k_{d l} a_{p l}(t) & 0 \\
0 & 0 & 0
\end{array}\right]}_{\breve{\mathbf{D}}_{O l}(t)} \underbrace{\left[\begin{array}{c}
q_{z l}\left(t-\tau_{l}\right) \\
\Delta h_{l}\left(t-\tau_{l}\right) \\
\Delta a_{p l}\left(t-\tau_{l}\right)
\end{array}\right]}_{\Delta \breve{\mathbf{w}}_{l}\left(t-\tau_{l}\right)},
\end{aligned}
$$

or, with the use of abbreviated notation:

$$
\breve{\mathbf{F}}_{l}(t)=\breve{\mathbf{F}}_{l}^{0}(t)-\breve{\mathbf{D}}_{P l}(t) \Delta \breve{\mathbf{w}}_{l}(t)+\breve{\mathbf{D}}_{O l}(t) \Delta \breve{\mathbf{w}}_{l}\left(t-\tau_{l}\right),
$$

where: $\breve{\mathbf{F}}_{l}(t)$ - vector of cutting forces of CE no. $l, \breve{\mathbf{F}}_{l}^{0}(t)-$ vector of cutting forces of CE no. $l$, resulted from the desired cutting geometry and kinematics, $\breve{\mathbf{D}}_{P l}(t)$ - matrix of linear proportional feedback interactions, $\breve{\mathbf{D}}_{O l}(t)$ - matrix of linear time-delayed feedback interactions, $\Delta \breve{\mathbf{w}}_{l}(t)$ - vector of deflections of CE no. $l$ at instant of time $t, \Delta \breve{\mathbf{w}}_{l}\left(t-\tau_{l}\right)$ - vector of deflections of CE no. $l$ at instant of time $t-\tau_{l}, q_{z l}(t)$ - relative displacement of edge and workpiece along direction $y_{l 1}$, at instant of time $t, q_{z l}\left(t-\tau_{l}\right)$ - relative displacement of edge and workpiece along direction $y_{l l}$, at instant of time $t-\tau_{l}$.

Vector (6) can be also described in six-dimensional space, i.e.:

$$
\mathbf{F}_{l}(t)=\mathbf{F}_{l}^{0}(t)-\mathbf{D}_{P l}(t) \Delta \mathbf{w}_{l}(t)+\mathbf{D}_{O l}(t) \Delta \mathbf{w}_{l}\left(t-\tau_{l}\right),
$$

where:

$$
\begin{gathered}
\mathbf{F}_{l}(t)=\operatorname{col}\left(\breve{\mathbf{F}}_{l}(t), \mathbf{0}_{3 \times 1}\right), \\
\Delta \mathbf{w}_{l}(.)=\operatorname{col}\left(\Delta \breve{\mathbf{w}}_{l}(.), \mathbf{0}_{3 \times 1}\right), \\
\mathbf{F}_{l}^{0}(t)=\operatorname{col}\left(\breve{\mathbf{F}}_{l}^{0}(t), \mathbf{0}_{3 \times 1}\right), \\
\mathbf{D}_{P l}(t)=\left[\begin{array}{cc}
\breve{\mathbf{D}}_{P l}(t) & \mathbf{0} \\
\mathbf{0} & \mathbf{0}
\end{array}\right]_{6 \times 6} \\
\mathbf{D}_{O l}(t)=\left[\begin{array}{cc}
\breve{\mathbf{D}}_{O l}(t) & \mathbf{0} \\
\mathbf{0} & \mathbf{0}
\end{array}\right]_{6 \times 6} .
\end{gathered}
$$


Cutting forces (5) are generated on the basis of the CEs' behaviour. The CEs are attached to appropriate nodes lying on the workpiece model surface, and - to the top of the bar element representing rotating tool (Fig. 1 and 2). Density of the used workpiece mesh is generally too small for representing the cutting process properly. Thus, geometric positions of the nearest surface nodes with respect to actual ball- end mill top position, for time instant $t$, were taken into account. Subsequently, workpiece vibration along direction perpendicular to its surface, at the current ball- end mill top position, has been determined. Dynamic change in the cutting layer thickness $\Delta h_{l}(t)$ was calculated by taking into account the slender ball -end mill vibration along $y_{12}$ direction of CE no. $l$. The reason was that vibration of the workpiece in its horizontal plane, was neglected. Dynamic change in the depth of cutting $\Delta a_{p l}(t)$ was calculated as relative displacement of the tool along $y_{l 3}$ direction and the workpiece along the direction perpendicular to the machined surface, at top position of ball end mill. Influence of the spindle speed on dynamics of the ball- end mill and on the other effects, was not included.

As a result of milling process modelling, a hybrid system which consists of separated subsystems, is obtained (Fig. 2). It consists of finite element method (FEM) model of workpiece and holder, i.e. stationary model of the one-side-supported flexible workpiece and the adjustable stiffness holder. The subsystem moves with the desired feed speed $v_{f}$ At the beginning the workpiece is idealised as a set of isoparametric 8-nodes finite elements (Kaliński, 2012). After making the modal transformation (Kaliński, 2012), behaviour of this subsystem is described by a vector of its modal coordinates a. Hence when we consider a finite number of normal modes mod of the subsystem, we shall define dynamic properties of it with the use of:

$\boldsymbol{\Omega}=\operatorname{diag}\left(\omega_{0 i}\right) \quad-\quad$ matrix of angular natural frequencies of the modal subsystem; $i=1, \ldots$, mod;

$\Psi=\left|\Psi_{1} \ldots \Psi_{\text {mod }}\right|-$ matrix of the considered mass normalised normal modes of the modal subsystem; $i=1, \ldots$, mod;

$\mathbf{Z}=\operatorname{diag}\left(\zeta_{i}\right) \quad-$ matrix of dimensionless damping coefficients of the modal subsystem; $\mathrm{i}=1, \ldots$, mod;

- tool FE model, i.e. non-stationary discrete model of ballend mill (the Euler-Bernoulli bar having local immovable coordinate system $x_{e 1}, x_{e 2}, x_{e 3}$ ) and the cutting process (i.e. coupling element (CE) no. $l$, placed in the instantaneous position of the "active" cutting edge (Kaliński and Galewski, 2015; Kaliński, 2012)). Cutting edges are considered "active" when they have contact with the workpiece, while the remaining ones are called "inactive". Behaviour of the subsystem is described by a vector of its generalised coordinates q. Dynamic properties of the Euler-Bernoulli bar are defined by the matrices of inertia, M, damping , L , and stiffness, K, (Kaliński, 2012);

- abstractive connective subsystem placed in the conventional contact point $S$ between the tool and the workpiece. Its generalised coordinates are related to the other ones by time-dependent constraint equations (Kaliński and Galewski, 2015; Kaliński, 2012).

Vector of deflections of CE no. $l$ is expressed as a function of vector of the generalised coordinates $\mathbf{q}$ and vector of the modal coordinates a. Hence we obtain the following relationship (Kaliński and Galewski, 2015; Kaliński, 2012):

$$
\Delta \mathbf{w}_{l}(t)=\left[\mathbf{T}_{l}(t)-\mathbf{W}_{l}(t)\right] \xi
$$

where:

$$
\mathbf{T}_{l}(t)=\boldsymbol{\Theta}_{l}(t) \mathbf{S}_{l}(t)
$$

- transformation matrix of the displacements' vector $\mathbf{q}$ from the coordinate system $x_{e 1}, x_{e 2}, x_{e 3}$ of the tool FE model to the coordinate system $y_{l 1}, y_{l 2}, y_{l 3}$ of CE no. $l$ (Kaliński and Galewski, 2015; Kaliński, 2012),

$$
\boldsymbol{\Theta}_{l}(t)=\left[\begin{array}{cc}
\breve{\boldsymbol{\Theta}}_{l}(t) & 0 \\
0 & \breve{\boldsymbol{\Theta}}_{l}(t)
\end{array}\right]
$$

- matrix of cosines of angles between the axes : $y_{l 1}, y_{l 2}, y_{l 3}$ of CE no. $l$, and $x_{e 1}, x_{e 2}, x_{e 3}$ of the tool FE model, whereas:

$$
\breve{\boldsymbol{\Theta}}_{l}(t)=\left[\begin{array}{ccc}
\cos \varphi_{l} t & -\sin \varphi_{l} t & 0 \\
\sin \varphi_{l}(t) & \cos \varphi_{l}(t) & 0 \\
0 & 0 & 1
\end{array}\right]
$$

$\mathbf{S}_{l}(t)$ - matrix of coordinates of attachment of CE no. $l$ to the tool FE model, in the coordinate system $x_{e 1}, x_{e 2}, x_{e 3}$ (Kaliński and Galewski, 2015; Kaliński, 2012);

$\mathbf{W}_{l}(t)$ - matrix of constraints between the modal displacements' vector a of the workpiece and holder model, and displacements in the coordinate system $y_{l 1}, y_{l 2}, y_{l 3}$ of CE no. $l$ (Kaliński and Galewski, 2015; Kaliński, 2012);

$\xi=\left\{\begin{array}{l}\mathbf{q} \\ \mathbf{a}\end{array}\right\}$ - vector of hybrid coordinates of the whole system.

After transformation of the vector of force interactions of CE no. $l(7)$ to hybrid coordinates, we obtained:

$$
\begin{aligned}
& {\left[\begin{array}{c}
\mathbf{T}_{l}^{T}(t) \\
-\mathbf{W}_{l}^{T}(t)
\end{array}\right] \mathbf{F}_{l}(t)=\left[\begin{array}{c}
\mathbf{T}_{l}^{T}(t) \\
-\mathbf{W}_{l}^{T}(t)
\end{array}\right] \mathbf{F}_{l}^{0}(t)+} \\
& +\left[\begin{array}{cc}
\mathbf{T}_{l}^{T}(t) \mathbf{D}_{P l}(t) \mathbf{T}_{l}(t) & -\mathbf{T}_{l}^{T}(t) \mathbf{D}_{P l}(t) \mathbf{W}_{l}(t) \\
-\mathbf{W}_{l}^{T} \mathbf{D}_{P l}(t) \mathbf{T}_{l}(t) & \mathbf{W}_{l}^{T}(t) \mathbf{D}_{P l}(t) \mathbf{W}_{l}(t)
\end{array}\right]\left\{\begin{array}{l}
\mathbf{q}_{s} \\
\mathbf{a}_{m}
\end{array}\right\}+ \\
& +\left[\begin{array}{c}
\mathbf{T}_{l}^{T}(t) \mathbf{D}_{O l}(t) \\
-\mathbf{W}_{l}^{T}(t) \mathbf{D}_{O l}(t)
\end{array}\right] \Delta \mathbf{w}_{l}\left(t-\tau_{l}\right) .
\end{aligned}
$$

As a result of the hybrid system's consideration, the matrix equation of dynamics of non-stationary model of the milling process in hybrid coordinates obtains the following form (Kaliński and Galewski, 2015; Kaliński, 2012): 


$$
\begin{aligned}
& {\left[\begin{array}{cc}
\mathbf{M} & \mathbf{0} \\
\mathbf{0} & \mathbf{I}
\end{array}\right] \ddot{\xi}+\left[\begin{array}{cc}
\mathbf{L} & \mathbf{0} \\
\mathbf{0} & 2 \mathbf{Z} \mathbf{\Omega}
\end{array}\right] \dot{\xi}+} \\
& +\left[\begin{array}{cc}
\mathbf{K}+\sum_{l=1}^{i_{l}} \mathbf{T}_{l}^{T}(t) \mathbf{D}_{P l}(t) \mathbf{T}_{l}(t) & -\sum_{l=1}^{i_{l}} \mathbf{T}_{l}^{T}(t) \mathbf{D}_{P l}(t) \mathbf{W}_{l}(t) \\
-\sum_{l=1}^{i_{l}} \mathbf{W}_{l}^{T}(t) \mathbf{D}_{P l}(t) \mathbf{T}_{l}(t) & \mathbf{\Omega}^{2}+\sum_{l=1}^{i_{l}} \mathbf{W}_{l}^{T}(t) \mathbf{D}_{P l}(t) \mathbf{W}_{l}(t)
\end{array}\right] \xi= \\
& = \\
& {\left[\begin{array}{c}
\sum_{l=1}^{i_{l}} \mathbf{T}_{l}^{T}(t) \mathbf{F}_{l}^{0}(t)+\mathbf{T}_{l}^{T}(t) \mathbf{D}_{O l}(t) \Delta \mathbf{w}\left(t-\tau_{l}\right) \\
-\sum_{l=1}^{i_{l}} \mathbf{W}_{l}^{T}(t) \mathbf{F}_{l}^{0}(t)+\mathbf{W}_{l}^{T}(t) \mathbf{D}_{O l}(t) \Delta \mathbf{w}\left(t-\tau_{l}\right)
\end{array}\right]}
\end{aligned}
$$

where $i_{l}$ is the number of „active” coupling elements.

In the particular case (Kaliński, 2012) when axis of the milling tool is perpendicular to the milled surface, the matrix of constraints, $\mathbf{W}_{l}(t)$, does not depend on current position of CE no. l. Thus we obtain:

$$
\mathbf{W}_{l}(t)=\mathbf{W}(t)=\mathbf{C}_{W} \hat{\mathbf{W}}(t) \boldsymbol{\Psi},
$$

where:

$$
\mathbf{C}_{W}=\operatorname{col}(0 \quad 0 \quad 100000)
$$

$\hat{\mathbf{W}}(t)$ - matrix of constraints between the modal displacements a, and vertical displacements of the workpiece, at current position of the conventional contact point $S$.

Now the matrix of Eq. 18 is simplified to following form:

$$
\begin{aligned}
& {\left[\begin{array}{cc}
\mathbf{M} & \mathbf{0} \\
\mathbf{0} & \mathbf{I}
\end{array}\right] \ddot{\xi}+\left[\begin{array}{cc}
\mathbf{L} & \mathbf{0} \\
\mathbf{0} & 2 \mathbf{Z} \boldsymbol{\Omega}
\end{array}\right] \dot{\xi}+} \\
& +\left[\begin{array}{cc}
\mathbf{K}+\sum_{l=1}^{i_{l}} \mathbf{T}_{l}^{T}(t) \mathbf{D}_{P l}(t) \mathbf{T}_{l}(t) & -\sum_{l=1}^{i_{l}} \mathbf{T}_{l}^{T}(t) \mathbf{D}_{P l}(t) \mathbf{W}(t) \\
\mathbf{0} & \mathbf{\Omega}^{2}
\end{array}\right] \boldsymbol{\xi}= \\
& =\left[\begin{array}{c}
\sum_{l=1}^{i_{l}} \mathbf{T}_{l}^{T}(t) \mathbf{F}_{l}^{0}(t)+\mathbf{T}_{l}^{T}(t) \mathbf{D}_{O l}(t) \Delta \mathbf{w}\left(t-\tau_{l}\right) \\
\mathbf{0}
\end{array}\right]
\end{aligned}
$$

On the basis of the above presented considerations, it is possible to show that in such particular case also instantaneous change in depth of cutting does not depend on current position of CE no. $l$. That is to say:

$$
\Delta a_{p l}(t)=\Delta a_{p}(t)=\hat{\mathbf{W}}(t) \mathbf{\Psi a} .
$$

\section{SIMULATIONS}

For the purpose of computer simulations of the milling process the holder with the workpiece and slender ball- end mill are idealised with the use of the FEM. Dynamic FE simulation is usual practice for acquiring knowledge about behaviour of the idealised real ? structures (Iwicki et al., 2014), or performance of real machining processes (Siemiątkowski and Przybylski, 2006). Rotating part of the workpiece holder is considered to be lumped mass supported by linear spring (Fig. 1). Appropriate degrees of freedom of the workpiece are fixed with the use of both Single Point Constraints and Multi Point Constraints, under assumption that deformability of the workpiece holder body was neglected. Slender ball-end mill is idealised by the Euler-Bernoulli bar. Its dimensions (i.e. length of $123 \mathrm{~mm}$, diameter of $16 \mathrm{~mm}$ ) and material properties are so selected as to represent dynamic properties of real ball-end mills that the authors have utilised before (i.e. having double bending mode at natural frequency of $743 \mathrm{~Hz}$ ). Discrete model of the workpiece ( of EN AW-6101A aluminium alloy material, mass density of $2.7 \times 10^{3} \mathrm{~kg} / \mathrm{m}^{3}$, Young modulus of $7 \times 10^{10} \mathrm{~Pa}$ ) consists of about 2800 eightnode isoparametric finite elements. The mesh resolution is about $2.5 \mathrm{~mm}$. The workpiece dimensions are $160 \mathrm{~mm} \times$ $50 \mathrm{~mm} \times 5 \mathrm{~mm}$. The FEM model of the workpiece was well correlated before with the real workpiece installed in a rigid workpiece holder, with the use of the experimental modal analysis techniques.

Even if the size of the model is not unexceptionally large, the number of required computer simulations makes it unusable in case of direct time-integration methods. Moreover direct time computation requires different damping models which could be at least partially obtained by means of the commonly used experimental modal analysis techniques (Euczak et al., 2014). Thus it is more convenient to use modal model instead and modal time synthesis method for the simulation of the cutting process.

Basing upon former authors' experience the frequency range for analysis is limited to $1000 \mathrm{~Hz}$. Within such frequency range four normal modes can be observed, i.e. two modes of workpiece holder with the workpiece and double mode of the slender ball-end mill (whose natural frequency is over 700 $\mathrm{Hz}$ ). Natural frequencies of the holder with the workpiece, for different workpiece holder spring settings, are presented in Tab. 1. Both of the modes for the holder with the workpiece are presented in Fig. 3 and 4 at the spring stiffness setting of $5600 \mathrm{~N} / \mathrm{mm}$. These modes do not change so much due to different spring stiffness settings of the workpiece holder. However both natural frequencies change due to adjustment of the spring stiffness. It was also noticed that both normal modes of the holder with the workpiece are well coupled with the workpiece. Any local modes' phenomena were not observed in this case. If we consider the latter, as well as effective mass properties of both modes, the first natural frequency can be expected to be a good approximation of the chatter frequency (see Eq. 1). 
Tab. 1. Natural frequencies of two first modes of variable stiffness holder with workpiece

\begin{tabular}{|c|c|c|}
\hline $\begin{array}{c}\text { Spring stiffness } \\
{[\mathrm{N} / \mathrm{mm}]}\end{array}$ & $\begin{array}{c}1^{\text {st }} \text { natural freq. } \\
{[\mathrm{Hz}]}\end{array}$ & $\begin{array}{c}2^{\text {nd }} \text { natural freq. } \\
{[\mathrm{Hz}]}\end{array}$ \\
\hline 14800 & 138.62 & 468.67 \\
\hline 11000 & 131.36 & 427.48 \\
\hline 8500 & 124.08 & 398.47 \\
\hline 6800 & 117.16 & 377.87 \\
\hline 5600 & 110.78 & 362.93 \\
\hline 4700 & 104.83 & 351.54 \\
\hline 4000 & 99.27 & 342.60 \\
\hline 3470 & 94.36 & 335.80 \\
\hline 3050 & 89.94 & 330.39 \\
\hline 2670 & 85.43 & 325.50 \\
\hline & & \\
\hline
\end{tabular}

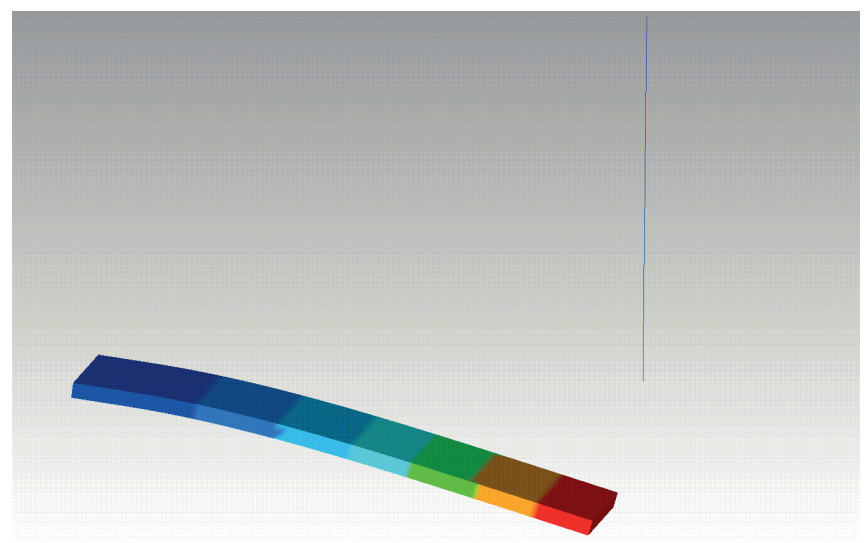

Fig. 3. First normal mode $(110.78 \mathrm{~Hz})$ of the stiffness holder with workpiece at spring stiffness of $5600 \mathrm{~N} / \mathrm{mm}$

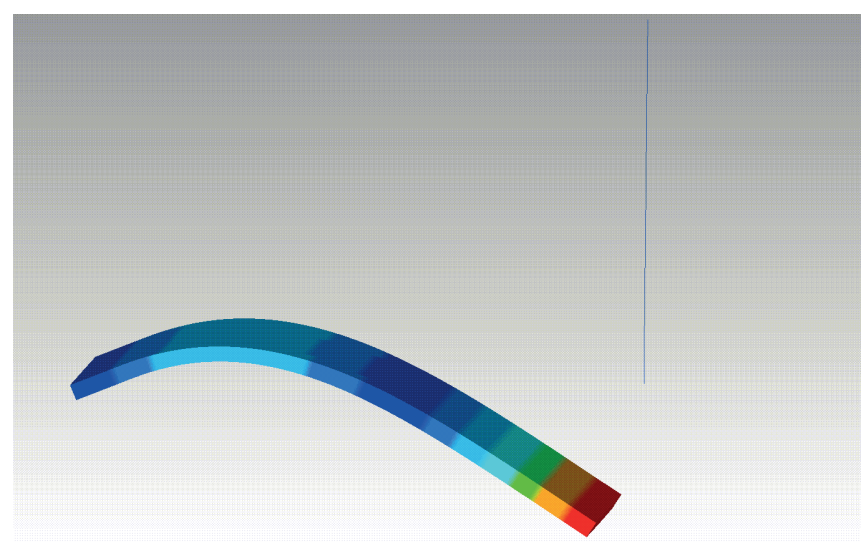

Fig. 4. Second normal mode $(362.93 \mathrm{~Hz})$ of the stiffness holder with workpiece at spring stiffness of $5600 \mathrm{~N} / \mathrm{mm}$
A series of simulations of the milling process performance was done on the basis of the determined matrix equation of dynamics (Eq. 21), and the adopted direct "step by step"integration Newmark method (Kaliński, 2001). Although it is possible to so adjust integration parameters as to make the method unconditionally stable, some calculation problems may occur when cutting edges lose contact with the workpiece (i.e. due to dynamic change in the cutting layer thickness $\Delta h_{l}(\mathrm{t})$ caused by high level of the observed vibrations). Therefore it is important to synchronize well integration time steps with the time delay $\tau_{l}$ between the same positions of subsequent tool cutting edges. In case of a fully developed chatter vibration the obtained results can be only used as an approximation of the milling process and thus conclusion in such case could only be whether the observed process is unstable [or stable?].

The results of simulation of the workpiece vertical vibration are obtained for a desired range of spindle speeds and adjusted values of the holder stiffness. The standard deviation values of displacements (Tab. 2) and relevant chatter amplitudes (Tab. 3) are determined. Standard deviation is used as a general index of vibration level and, in fact, represents the RMS value of displacements but calculated with respect to non-zero mean displacement value. It is worth noticing that application of the adjustable stiffness holder reflects good performance in scope of chatter reduction within the useful range of spindle speeds from 13000 to $16700 \mathrm{rev} / \mathrm{min}$. In majority of simulations expected optimum combinations of "spindle speed-holder spring stiffness" are obtained (Tab. 3).

In the case of simulation at the spindle speed $n=15745$ $\mathrm{rev} / \mathrm{min}$ and the optimum holder stiffness $k_{\mathrm{s}}=11000 \mathrm{~N} / \mathrm{mm}$, vertical displacements of the workpiece vibration (Fig. 5a) and their amplitude spectra (Fig. 5b) are presented. It is clearly visible that vibration level was low and the chatter did not occur (there is no spectrum peak in vicinity of the workpiece natural frequency). In this case vibration level slightly exceeded $0.01 \mathrm{~mm}$, which is about $10 \%$ of the nominal depth of cutting. 
Tab. 2. Standard deviation of displacements [ $\mathrm{mm}]$ ( expected optimum pairs are distinguished with grey background)

\begin{tabular}{|c|c|c|c|c|c|c|c|}
\hline \multirow{2}{*}{$\begin{array}{l}\text { Spindle speed } \\
{[\mathrm{rev} / \mathrm{min}]}\end{array}$} & \multicolumn{7}{|c|}{ Holder spring stiffness $[\mathrm{N} / \mathrm{mm}]$} \\
\hline & 14800 & 11000 & 8500 & 6800 & 5600 & 4700 & 4000 \\
\hline 17651 & 0.002366 & & & & & & \\
\hline 16745 & & 0.007657 & & & & & \\
\hline 16651 & 0.002539 & 0.005380 & & & & & \\
\hline 15869 & & & 0.002711 & & & & \\
\hline 15745 & 0.003676 & 0.002613 & 0.002671 & 0.002523 & 0.002193 & & \\
\hline 15651 & 0.003866 & & & & & & \\
\hline 15284 & & & & & 0.002547 & & \\
\hline 15047 & & & & 0.002860 & & & \\
\hline 14869 & & 0.004322 & 0.002926 & 0.002787 & 0.002927 & & \\
\hline 14745 & & 0.004957 & & & & & \\
\hline 14284 & & & & & 0.003170 & & \\
\hline 14047 & & & 0.006864 & 0.003431 & 0.003163 & & \\
\hline 13869 & & & 0.008736 & & & & \\
\hline 13784 & & & & & 0.003831 & & \\
\hline 13581 & & & & & & 0.003441 & \\
\hline 13284 & & & & 0.008378 & 0.004034 & 0.004537 & 0.003627 \\
\hline 13047 & & & & 0.010622 & & & \\
\hline 12581 & & & & & 0.015531 & 0.020466 & 0.031396 \\
\hline 12284 & & & & & 0.024176 & & \\
\hline 11932 & & & & & & 0.059660 & 0.059596 \\
\hline 11581 & & & & & & 0.078220 & \\
\hline
\end{tabular}

Tab. 3. Vibration amplitude of the $1^{\text {st }}$ mode $[\mathrm{mm}]$ (expected optimum pairs are distinguished with grey background and bold numbers)

\begin{tabular}{|c|c|c|c|c|c|c|c|}
\hline \multirow{2}{*}{$\begin{array}{l}\text { Spindle speed } \\
\text { [rev/min] }\end{array}$} & \multicolumn{7}{|c|}{ Holder spring stiffness $[\mathrm{N} / \mathrm{mm}]$} \\
\hline & 14800 & 11000 & 8500 & 6800 & 5600 & 4700 & 4000 \\
\hline 16745 & & 0.007286 & & & & & \\
\hline 16651 & 0.000310 & 0.004502 & & & & & \\
\hline 15869 & & & 0.001615 & & & & \\
\hline 15745 & 0.001045 & 0.000317 & 0.001557 & 0.001632 & 0.000666 & & \\
\hline 15651 & 0.001282 & & & & & & \\
\hline 15284 & & & & & 0.000942 & & \\
\hline 15047 & & & & 0.001510 & & & \\
\hline 14869 & & 0.002834 & 0.000472 & 0.000848 & 0.001501 & & \\
\hline 14745 & & 0.003316 & & & & & \\
\hline 14284 & & & & & 0.001103 & & \\
\hline 14047 & & & 0.004760 & 0.000747 & 0.005021 & & \\
\hline 13869 & & & 0.006904 & & & & \\
\hline 13784 & & & & & 0.001447 & & \\
\hline 13581 & & & & & & 0.000357 & \\
\hline 13284 & & & & 0.006728 & 0.000521 & 0.002755 & 0.002057 \\
\hline 13047 & & & & 0.008114 & & & \\
\hline 12581 & & & & & 0.005560 & 0.021472 & 0.033184 \\
\hline 12284 & & & & & 0.029790 & & \\
\hline 11932 & & & & & & 0.074891 & 0.073382 \\
\hline 11581 & & & & & & 0.082545 & \\
\hline
\end{tabular}


a)

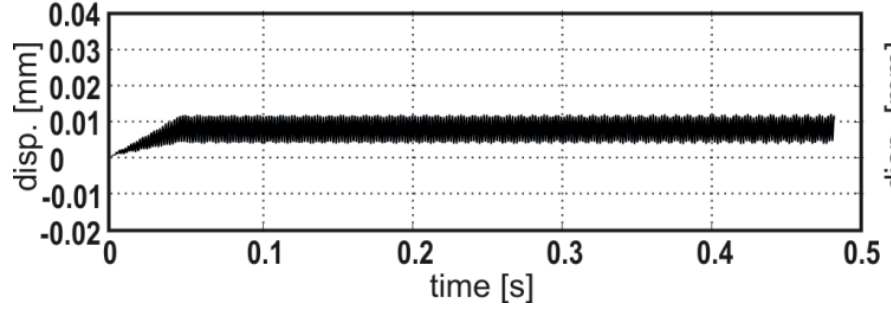

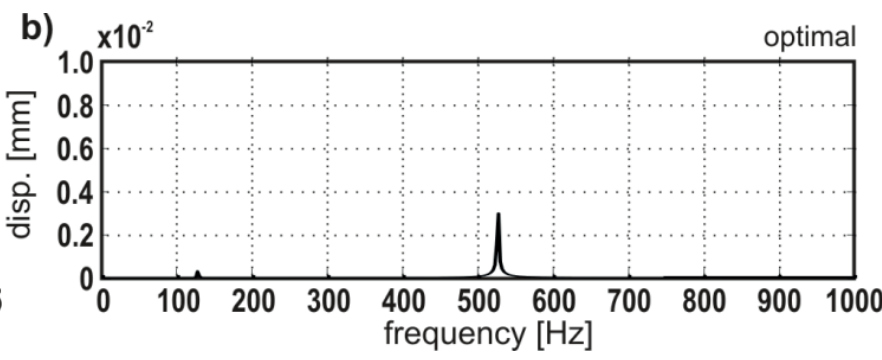

Fig 5. Displacement, (a), and its spectrum, (b), for the optimum pair of the spindle speed $n=15745 \mathrm{rev} / \mathrm{min}$ and the holder stiffness of $11000 \mathrm{~N} / \mathrm{mm}$

a)
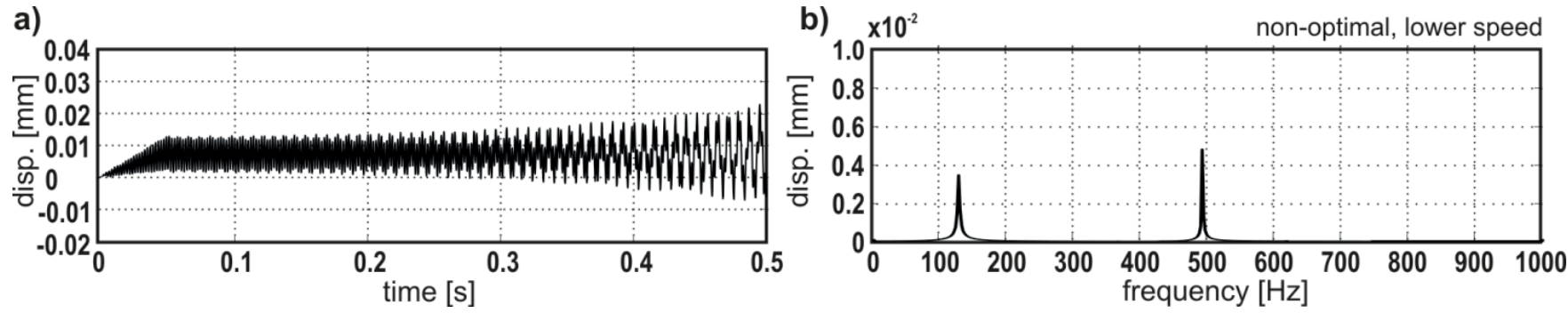

Fig 6. Displacement, (a), and its spectrum, (b), for the non-optimum pair of the spindle speed $n=14745 \mathrm{rev} / \mathrm{min}$ and the holder stiffness of $11000 \mathrm{~N} / \mathrm{mm}$

a)

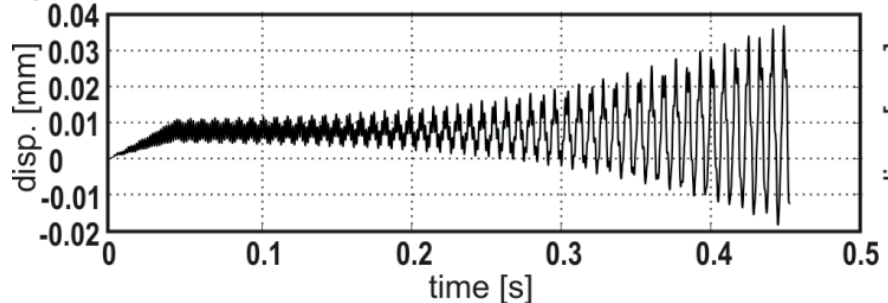

b)

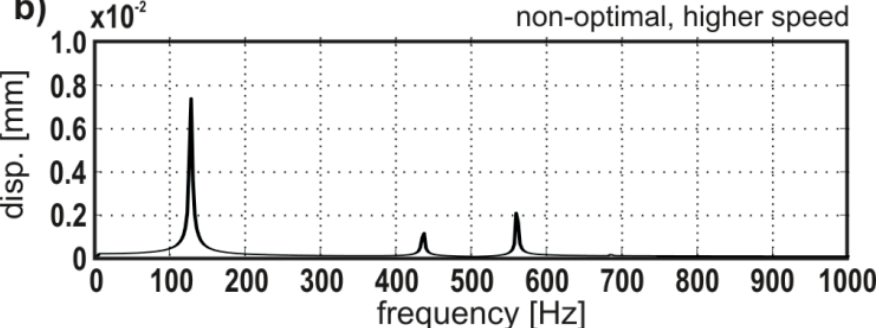

Fig 7. Displacement, (a), and its spectrum, (b), for the non-optimum pair of the spindle speed $n=16745 \mathrm{rev} / \mathrm{min}$ and the holder stiffness of $11000 \mathrm{~N} / \mathrm{mm}$

a)

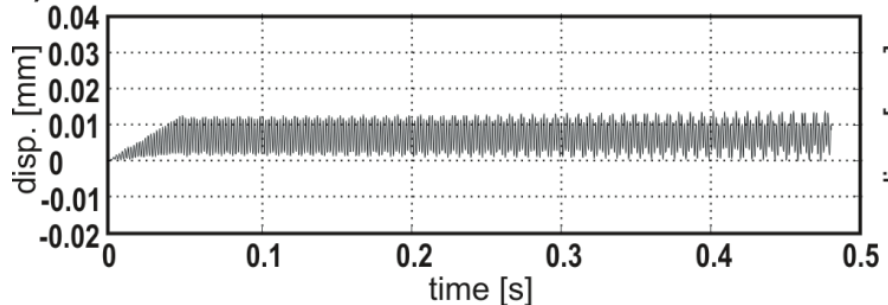

b)

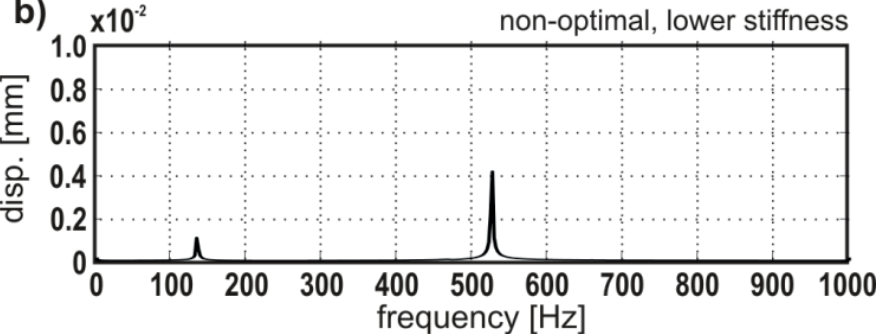

Fig 8. Displacement, (a), and its spectrum, (b) , for the non-optimum pair of the spindle speed $n=15745 \mathrm{rev} / \mathrm{min}$ and the holder stiffness of $8500 \mathrm{~N} / \mathrm{mm}$

a)

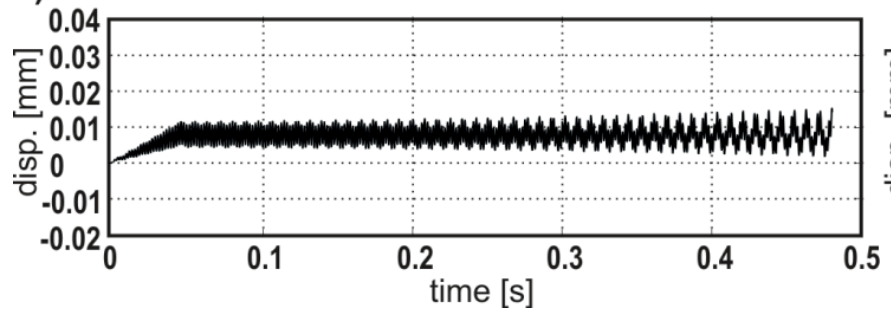

b)

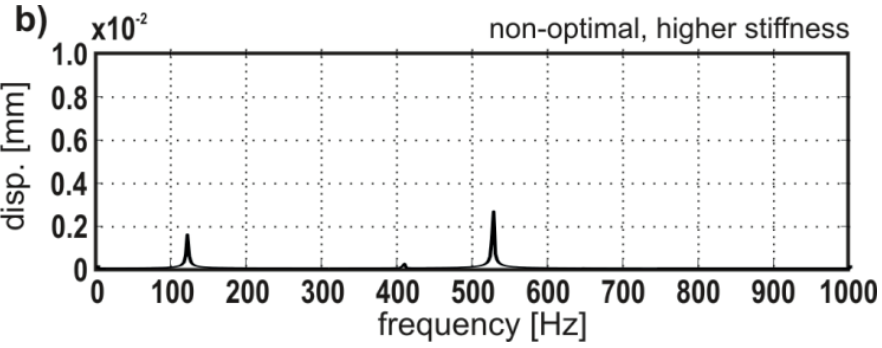

Fig 9. Displacement, (a), and its spectrum, (b), for the non-optimum pair of the spindle speed $n=15745 \mathrm{rev} / \mathrm{min}$ and the holder stiffness of $14800 \mathrm{~N} / \mathrm{mm}$ 
In the relevant amplitude spectrum only one harmonics concerning frequency of entering cutting edges into the workpiece (i.e. that of $525 \mathrm{~Hz}$ ), is observed. In the case of non-optimum spindle speeds, vibration level approaches either over $0.02 \mathrm{~mm}$ (Fig. 6a, at lower speed) or even over $0.03 \mathrm{~mm}$ (Fig. 7a, at higher speed). The relevant frequency plots (Fig. 6b and 7b) illustrate, except harmonics concerning appropriate spindle speeds of 14745 and $16745 \mathrm{rev} / \mathrm{min}$, appearance of strong chatter amplitudes whose frequency is close to the first natural frequency equal to $131.36 \mathrm{~Hz}$ of the holder with the workpiece (Tab. 1). Additionally, change of the holder stiffness, with respect to its optimum value, causes a noticeable increase in vibration level, as well as significant chatter amplitudes (Fig. 8 and 9).

\section{CONCLUSIONS}

Results of the simulations showed that to modify the workpiece dynamic properties is possible with the use of the proposed new workpiece holder. The simulations for different pairs of holder stiffness and spindle speed show that vibrations are the lowest only in case of a proper optimum combination of the two parameters. These results mean that the proposed idea of a variable stiffness holder has a great potential. In case of a standard perfectly rigid holder, a method for vibration reduction was to choose the proper spindle speed on the basis of the Liao-Young condition. However the determined optimum spindle speed might not be optimum from other points of view (i.e. technological ones). The proposed variable stiffness holder allows to adjust workpiece's dynamic properties in such a way that an arbitrarily given spindle speed should accomplish the generalised Liao-Young condition for the desired operational spindle speeds of the milling machine. However vibration surveillance efficiency is really evident only in case of some values of spindle speeds. Hence the proposed approach should be subjected to further investigations. Despite the latter, the obtained results may serve as a basis for the optimum performance planning of machining processes (Deja and Siemiątkowski, 2013).

\section{REFERENCES}

1. Brecher C., Manoharan D., Ladra U., Köpken H.-G.: Chatter suppression with an active workpiece holder. Production Engineering Research and Development, Vol. 4 (2010), pp.239-245.

2. Deja M., Siemiątkowski M. S.: Feature-based generation of machining process plans for optimised parts manufacture, , August 2013, Vol. 24, Issue 4, pp. 831-846.

3. Dohner J. L., Lauffer J. P., Hinnerichs T. D., Shankar N., Regelbrugge M., Kwan C.-M, Xu R., Winterbauer B., Bridgerf K. : Mitigation of chatter instabilities in milling by active structural control. Journal of Sound and Vibration, Vol. 269(2004), pp. 197-211.

4. Ganguli A., Deraemaekar A., Horodinca M., Preumont A.: Active damping of chatter in machine tools - demonstration with a "Hardware in the Loop" Simulator. Proceedings of the Institution of Mechanical Engineers, Part I: Journal of System Control Engineering, Vol. 219 (2005), pp. 359-369.

5. Hoffmann M., Powałka B., Berczyński S., Pajor M.: Identification of cutting forces in frequency domain in milling. Advances in Manufacturing Science and Technology, Vol. 34 (1) (2010), pp. 5-20.

6. Iwicki P., Tejchman A., Chróścielewski J. : Dynamic FE simulations of buckling process in thin-walled cylindrical metal silos. Thin-Walled Structures, (2014), pp. 344-359.

7. Kaliński K. J.: Vibration surveillance of mechanical systems which are idealised discretely (in Polish). Gdańsk University of Technology Publishers, Gdańsk 2001.

8. Kaliński K. J. A surveillance of dynamic processes in mechanical systems (in Polish). Gdańsk University of Technology Publishers, Gdańsk 2012.

9. Kaliński K. J., Chodnicki M., Mazur M., Galewski M. A.: Vibration surveillance system with variable stiffness holder for flexible details milling.[in:] Applied Non-Linear Dynamical Systems, Springer Proceedings in Mathematics and Statistics, J. Awrejcewicz, Vol. 93(2014b), pp. 175-184.

10. Kaliński K. J., Chodnicki M., Galewski M. A., Mazur M. : Vibration Surveillance for Efficient Milling of Flexible Details in Adjustable Stiffness Holder.Vibroengineering Procedia, Vol. 3 (2014c), pp. 215-218.

11. Kaliński K. J., Galewski M. A. : Chatter vibration surveillance by the optimal-linear spindle speed control. Mechanical Systems and Signal Processing, Vol. 25 (2011), pp. 383-399.

12. Kaliński K. J., Galewski M. A.: Vibration surveillance supported by Hardware-In-the-Loop Simulation in milling flexible workpieces. Mechatronics, Vol. 24(2014), pp. 1071-1082.

13. Kaliński K. J., Galewski M. A. : Optimal Spindle Speed Determination for Vibration Reduction During BallEnd Milling of Flexible Details. International Journal of Machine Tools and Manufacture, Vol. 92 (2015), pp. 19-30.

14. Kaliński K. J., Galewski M. A., Mazur M.: High Speed Milling vibration surveillance with optimal spindle speed 
based on optimal speeds map. Key Engineering Materials, Vol. 597(2014a), pp.125-130.

15. Kaliński K. J., Mazur M., Galewski M. A. : High speed milling vibration surveillance with the use of the map of optimal spindle speeds. [in:] Proceedings of the 8th International Conference on High Speed Machining, Metz, France, (2010), pp. 300-305.

16. Kaliński K. J., Mazur M., Galewski M. A.: The Optimal Spindle Speed Map for Reduction of Chatter Vibration During Milling of Bow Thruster Blade. Solid State Phenomena, Vol. 198 (2013), pp. 686-691.

17. Kim M.H., Won D., Ziegert J. : Numerical Analysis and Parameter Study of a Mechanical Damper for Use in Long Slender Endmills. International Journal of Machine Tools and Manufacture, Vol. 46 (2006), pp. 500-507.

18. Liao Y.S., Young Y.C.: A new on-line spindle speed regulation strategy for chatter control. International Journal of Machine Tools and Manufacture, Vol. 36 (1996), pp. 651-660.

19. Łuczak M., Manzato S., Peeters B., Branner K., Berring P., Kahsin M. : Updating Finite Element Model of a Wind Turbine Blade Section Using Experimental Modal Analysis Results, Shock and Vibration, Vol. 2014, issue 1, pp. 71-82.

20. Moradi H., Vossoughi G., Movahhedy M.R., Salarieh H.: Suppression of nonlinear regenerative chatter in milling process via robust optimal control. Journal of Process Control, Vol. 23 (2013), pp. 631-648.

21. Nouari M., List G., Girot F. : Wear mechanisms in dry machining of aluminium alloys. International Journal of Mechanical Production Systems Engineering, Vol. 4 (2003) , pp. 22-29

22. Pajor M., Hoffmann M., Marchelek K. : Identification of cutting process model parameters for multi-edge rotating tools (in Polish), Modelowanie Inżynierskie, Vol. 10 (2011) , pp. 307-314.

23. Parus A., Pajor M., Hoffmann M. : Suppression of SelfExcited Vibration in Cutting Process Using Piezoelectric and Electromagnetic Actuators. Advances in Manufacturing Science and Technology, Vol. 33 (2009), pp. 35-50.

24. Powałka B., Pajor M., Berczyński S. : Identification of nonlinear cutting process model in turning. Advances in Manufacturing Science and Technology, Vol. 33 (3) (2009), pp. 17-25.

25. Quintana G., Ciurana J. : Chatter in machining processes: a review. International Journal of Machine Tools and Manufacture, Vol. 51(2011), pp. 363-376.
26. Rashid M.K. : Simulation Study on the Improvements of Machining Accuracy by Using Smart Materials. Robotics and Computer-Integrated Manufacturing, Vol. 21(2005), pp. 249-257.

27. Sellmeier V., Denkena B.: High speed process damping in milling. CIRP Journal of Manufacturing Science and Technology, Vol. 5 (2012), pp. 8-19.

28. Siemiątkowski M., Przybylski W. : Simulation studies of process flow with in-line part inspection in machining cells. Journal of Materials Processing Technology, January 10, 2006, Vol. 171, Issue 1, pp. 27-34.

29. Soliman E., Ismail F. : Chatter suppression by adaptive speed modulation. International Journal of Machine Tools and Manufacture, Vol. 37(1997) , pp. 355-369.

30. Tomków J. : Vibrostability of machine tools (in Polish), The Scientific and Technical Publications, Warsaw 1997. Plik : PMR-spec_KK_M..: 33256 zn. norm. [18,5 str], stan 2017-02-06, kor. epw

\section{CONTACT WITH THE AUTHORS}

Krzysztof J. Kaliński

e-mail:kkalinsk@o2.pl

\section{Marek A. Galewski}

e-mail:margalew@pg.gda.pl

Michał Mazur

e-mail:mazur.m.r@gmail.com

\section{Marek Chodnicki}

e-mail:marek@chodnicki.pl

Faculty of Mechanical Engineering Gdańsk University of Technology ul. G. Narutowicza 11/12, 80-233 Gdańsk

Poland 